\title{
Simulation of Three-Dimensional Dusty Plasmas
}

\author{
Bin Liu and John Goree
}

\begin{abstract}
The structure and dynamics of dust particles in a 3-D dusty plasma is characterized using a Langevin molecular dynamics simulation with a Yukawa potential. Conditions are set appropriate for a liquid-like strongly coupled plasma. The positions of dust particles are shown in an image. The thermal motion of particles is decomposed into the longitudinal wave spectrum, showing a distinctive dispersion relation.
\end{abstract}

Index Terms-Dusty plasmas, liquids, numerical simulation, waves.

$\mathbf{W}$ E USE the Langevin dynamics simulation method of [1] to perform new simulations to characterize structure and waves in a 3-D dusty plasma. The equation of motion

$$
m_{p} \ddot{\mathbf{r}}_{j}=-v_{g} m_{p} \dot{\mathbf{r}}_{j}+\zeta_{g j}(t)-\nabla \sum_{l} \phi_{j l}-\nabla \Phi
$$

for the $j$ th particle includes electrostatic forces due to particleparticle interaction $\phi_{j l}$, a confinement potential $\Phi$, gas friction $-v_{g} m_{p} \dot{\mathbf{r}}_{j}$, and random Brownian force $\zeta_{g j}$ due to gas molecules. Here, $m_{p}$ is the particle mass and $v_{g}$ is the gas friction constant. We model the interparticle potential as a pairwise Yukawa potential, $\phi_{j l}\left(r_{j l}\right)=\left(Q^{2} / 4 \pi \varepsilon_{0} r_{j l}\right) e^{-r_{j l} / \lambda_{D}}$ for identical dust particles of charge $Q$ with a screening length $\lambda_{D}$ due to the electrons and ions. The collection of dust particles can be characterized by two dimensionless parameters: the Coulomb coupling parameter $\Gamma=Q^{2} / 4 \pi \varepsilon_{0} a k_{\mathrm{B}} T_{\mathrm{d}}$ and the screening parameter $\kappa=a / \lambda_{D}$. Here $a=\left(3 / 4 \pi n_{\mathrm{d}}\right)^{1 / 3}$ is the Wigner-Seitz radius, $n_{\mathrm{d}}$ is the number density of dust particles, and $T_{\mathrm{d}}$ is the particle kinetic temperature. For the coupling parameter $\Gamma>1$, the dust component is said to be strongly coupled, and dust particles can self-organize like atoms in a solid or liquid and sustain waves [2], [3].

Our simulation parameters are for the PK-4 instrument [4]. We use microsphere dust particles of radius $3.43 \mu \mathrm{m}$ and $m_{p}=2.55 \times 10^{-13} \mathrm{~kg}$, with neon gas at $50 \mathrm{~Pa}$ pressure and $0.03 \mathrm{eV}$ temperature so that $\nu_{g}=51 \mathrm{~s}^{-1}$. We assume $Q=-8520 e, n_{\mathrm{d}}=3 \times 10^{4} \mathrm{~cm}^{-3}$, and $\lambda_{D}=8.3 \times 10^{-3} \mathrm{~cm}$. The characteristic interparticle distance is $a=0.020 \mathrm{~cm}$, so that $\kappa=2.4$. The characteristic time for particle motion is $\omega_{p}=157 \mathrm{rad} / \mathrm{s}$, where $\omega_{p}=\left(Q^{2} n_{\mathrm{d}} / \varepsilon_{0} m_{p}\right)^{1 / 2}$.

We chose $T_{\mathrm{d}}=8.3 \mathrm{eV}$, corresponding to $\Gamma \approx 63$. For these values of $\Gamma$ and $\kappa$, the collection of dust particles is predicted

Manuscript received November 21, 2013; accepted April 19, 2014. Date of publication May 13, 2014; date of current version October 21, 2014. This work was supported in part by the National Aeronautics and Space Administration and in part by the National Science Foundation.

The authors are with the Department of Physics and Astronomy, University of Iowa, Iowa City, IA 52242 USA (e-mail: bin-liu@uiowa.edu; john-goree@uiowa.edu).

Color versions of one or more of the figures in this paper are available online at http://ieeexplore.ieee.org.

Digital Object Identifier 10.1109/TPS.2014.2321324 to behave like a liquid, according to the phase diagram of the Yukawa system [5]. We simulate $N=12800$ particles in a 3-D rectangular volume defined by a flat-bottomed confining potential $\Phi$.

Results shown in Fig. 1(a) reveal the structural arrangement of the dust particles at a time during the simulation. This image was prepared by plotting the simulated particles in a 3-D coordinate system, with a sphere representing each particle. In this structure, each particle is in a cage defined by its nearest neighbors, but the structure is irregular, not crystalline. A video can be seen at [6] showing similar particles as shown in Fig. 1(a) from a rotating viewpoint.

To quantify the order of the 3-D structure, we calculate the pair correlation function $g(r)$ [7], [8]. For this liquid, $g(r)$ has only one distinctive peak in Fig. 1(b), indicating short-range translational order.

We characterize the dynamics using a wave spectrum. We start by using the particle position $\mathbf{r}_{j}(t)$ and velocity $\dot{\mathbf{r}}_{j}(t)$ to calculate the time series of the so-called longitudinal current, for a specified wave vector $\mathbf{k}$

$$
J_{L}(k, t)=N^{-1} \sum_{j=1}^{N}\left[\dot{\mathbf{r}}_{j}(t) \cdot \mathbf{k} /|\mathbf{k}|\right] \exp \left[i \mathbf{r}_{j}(t) \cdot \mathbf{k}\right] .
$$

The spectral power $\left|J_{L}(k, \omega)\right|^{2}$ is then computed as the square modulus of the Fourier transformation in time of $J_{L}(k, t)$.

Results in Fig. 1(c) show that, as expected, the spectral power is concentrated along a curved band. The band has a great width in the $\omega-k$ space due to damping, arising from gas friction and the viscous motion of dust particles.

The band of spectral power in Fig. 1(c) corresponds to a real dispersion relation curve, which we plot in Fig. 1(c) as a dotted line. We determined this dispersion curve as the peak of the spectral power $\left|J_{L}(k, \omega)\right|^{2}$; to reduce the uncertainty, we computed $\omega$ for the peak as the first moment of the spectral power $\left|J_{L}(k, \omega)\right|^{2}$ for each value of $k$. The dispersion relation begins near $k a=0$ with an acoustic-like upward slope, that is, the wave is forward for long wavelengths. This upward trend reverses for $2<k a<4$, where the dispersion relation curve has a slightly negative slope, that is, the wave is backward.

\section{REFERENCES}

[1] J. Goree, B. Liu, and Y. Feng, "Diagnostics for transport phenomena in strongly coupled dusty plasmas," Plasma Phys. Controlled Fusion, vol. 55, no. 12, p. 124004, 2013.

[2] Z. Donkó, G. J. Kalman, and P. Hartmann, "Dynamical correlations and collective excitations of Yukawa liquids," J. Phys., Condensed Matter, vol. 20, no. 41, p. 413101, 2008.

[3] G. Kalman, M. Rosenberg, and H. E. DeWitt, "Collective modes in strongly correlated Yukawa liquids: Waves in dusty plasmas," Phys. Rev. Lett., vol. 84, no. 26, pp. 6030-6033, Jun. 2000. 

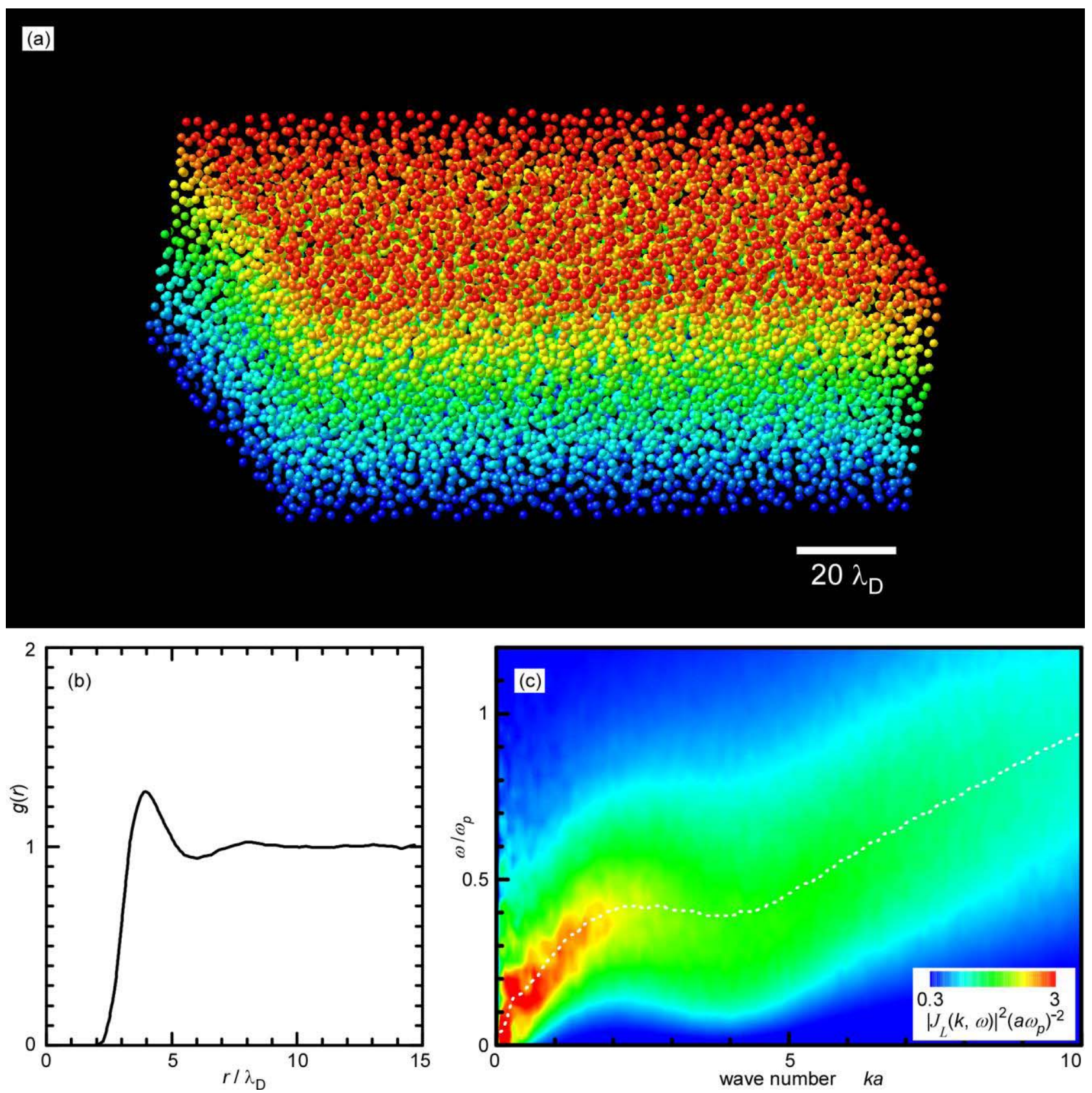

Fig. 1. (a) Structural arrangement of dust particles at a time during a 3-D Yukawa simulation. For clarity, the diameter of the dust particle microsphere is exaggerated here. Color indicates the height above the bottom plane. (b) Pair correlation function $g(r)$, indicating the disorder in the structural arrangement. (c) Wave spectrum for longitudinal motion, computed from particle positions and velocities. Color indicates spectral power, which is not smoothed; the results here have finite noise, which was reduced by averaging over eight runs of the simulation and 750 directions of $\mathbf{k}$. Dotted curve: real dispersion relation, as obtained from the weighted peak of the spectral power with respect to frequency.

[4] V. Fortov et al., "The project 'plasmakristall-4' (PK-4)—A new stage in investigations of dusty plasmas under microgravity conditions: First results and future plans," Plasma Phys. Controlled Fusion, vol. 47, no. 12B, pp. B537-B549, 2005.

[5] S. Hamaguchi, R. T. Farouki, and D. H. E. Dubin, "Triple point of Yukawa systems," Phys. Rev. E, vol. 56, no. 4, pp. 4671-4682, 1997.

[6] B. Liu and J. Goree. (2013, Nov.). Movie Showing the Structure of a Dusty Plasma [Online]. Available: http://ir.uiowa.edu/physics_pubs/1/
[7] J. P. Hansen and I. R. McDonald, Theory of Simple Liquids, 2nd ed. San Diego, CA, USA: Academic, 1986.

[8] T. S. Ramazanov, K. N. Dzhumagulova, A. N. Jumabekov, and M. K. Dosbolayev, "Structural properties of dusty plasma in direct current and radio frequency gas discharges," Phys. Plasmas, vol. 15, no. 5 , p. 053704,2008 